\title{
The effect of inhomogeneous water on seismic imaging in deepwater areas of the South China Sea
}

\author{
$\mathrm{JI} \mathrm{LiLi}^{1}$, LIN Mian $^{2 *} \&$ HAO TianYao ${ }^{1}$ \\ ${ }^{1}$ Institute of Geology and Geophysics, Chinese Academy of Sciences, Beijing 100029, China; \\ ${ }^{2}$ Institute of Mechanics, Chinese Academy of Sciences, Beijing 100190, China
}

Received January 9, 2013; accepted May 13, 2013; published online July 25, 2013

\begin{abstract}
This paper examines the effect of inhomogeneous water on seismic imaging in deep water areas. An appropriate partial differential equation is derived for the acoustic pressure field in inhomogeneous water, including current effects. Seismic wavefields are simulated and the results show that the traveltime of seismic waves can be affected. The maximum traveltime perturbation at zero offset is $20 \mathrm{~ms}$. In particular, the structure of horizontal reflectors below the water is distorted by mesoscale eddies. Variations of water temperature are the fundamental cause of the distortion. Finally, a calibration method for the distortion of seismic imaging caused by inhomogeneous water is presented.
\end{abstract}

inhomogeneous water, mesoscale eddies, distortion of seismic imaging, temperature perturbation

Citation: Ji L L, Lin M, Hao T Y. The effect of inhomogeneous water on seismic imaging in deepwater areas of the South China Sea. Chin Sci Bull, 2013, 58: 4443-4449, doi: 10.1007/s11434-013-5975-z

Seismic waves pass easily through shallow water (tens of meters deep), such that the water is taken as homogeneous during seismic data processing. However, in deep water (hundreds of meters deep), the question arises of whether the heterogeneity of the water can still be neglected. Mesoscale structures of the sea, such as eddies, fronts and currents, can be imaged through processing the seismic reflection data [1-6]. Moreover, the complicated set of interbed multiples, induced by the inhomogeneous water, can easily obscure primary reflections from relatively weak sedimentary reflectors. In the 1980s, Gonella \& Michon [7] and Phillips \& Dean [8] found that the oceanic fine structure could be mapped by analysis of a seismic data set; however, their work received little attention. In the beginning of this century, Holbrook et al. [9] discovered from three seismic sections near the Gorringe Ridge that imaged reflectors correspond to oceanic thermal structures, and that the characteristics of seismic reflections vary with different water masses. Subsequently, several studies in seismic oceanog-

*Corresponding author (email: linmian@imech.ac.cn) raphy have shown that the interface between the upper warm current and lower cold water in the Norwegian Sea can produce strong reflections [10]; that the amplitude of reflections from the boundary between the warm Kuroshio current and the cold Oyashio water changes markedly [11]; and that the reflectors in the upper and lower parts of mesoscale eddies are complicated [12]. The above research demonstrates that the heterogeneity of the water can be reflected in the seismic data and that the instability of the dynamic environment in inhomogeneous water can make the propagation of seismic waves more complicated. Thus, a new problem is identified in the processing of seismic data.

Since 2009, with the support of the National Natural Science Foundation of China and the National Basic Research Program of China, we have studied the following issues: (1) To what degree is seismic imaging influenced by inhomogeneous water? (2) What causes this influence? (3) How can the influence of the inhomogeneous water be eliminated from seismic data? The above three problems will be answered in this paper. Inhomogeneous water can be classified broadly into two categories: static water (a vertically 
stratified fluid in hydrostatic balance) and dynamic water (the hydrostatic balance of water is destroyed by an external disturbance, such as internal waves, mesoscale eddies and ocean fronts, etc.). The density and sound speed in static water is inhomogeneous. Although the forms of movement of the ocean are diverse, they can be described by the density field, the sound speed field and the current field, which vary greatly both spatially and temporally. Because of the special geography, monsoons, asymmetric heat and buoyancy, cold- and warm-core eddies can be observed frequently in deep areas of the South China Sea [13,14]. Compared with other mesoscale structures of the ocean, mesoscale eddies have characteristics of a long life cycle, a wide scope of influence and fast rotational speed. Additionally, the density, sound speed and current of mesoscale eddies vary greatly with space and time change. All this demonstrates that mesoscale eddies are a typical kind of dynamic water. Thus, this paper considers cold- and warm-core eddies as examples for studying the effect of dynamic water on seismic imaging and for analyzing the mechanism of distortion of seismic imaging, and finally concludes with general results.

\section{To what degree is a seismic image influenced by inhomogeneous water?}

First, an appropriate partial differential equation is derived for the acoustic pressure field in inhomogeneous water, including current effects [15].

$$
\widehat{c}_{w}{ }^{2} \hat{\rho}_{0} \widehat{\nabla} \cdot\left(\frac{1}{\hat{\rho}_{0}} \widehat{\nabla} \hat{p}_{1}\right)=2 \widehat{u}_{0} \frac{\partial^{2} \hat{p}_{1}}{\partial \widehat{t} \partial \hat{x}}+\frac{\partial^{2} \hat{p}_{1}}{\partial \widehat{t}^{2}} .
$$

Compared with the wave equation usually used in seismic modeling, eq. (1) has a term including the current effect $\left(\widehat{u}_{0}\right)$. It can be obtained from eq. (1) that the heterogeneity of the water is expressed by the density $\hat{\rho}_{0}$, the speed of sound $\hat{c}_{w}$ and the current $\hat{u}_{0}$. It is worthwhile to emphasize that, to observe the reflections yielded by the inhomogeneous water clearly, spurious numerical reflections at the lateral PML boundaries must be at least one order smaller than the magnitude of the physical phenomena of interest. Thus, the first-order PML introduced by Kormann et al. [16] is used in this paper. Our physical model is depicted in Figure 1(a). The water depth is $2200 \mathrm{~m}$, below which there are four homogeneous layers separated by flat interfaces: $\mathrm{H}_{1}, \mathrm{H}_{2}, \mathrm{H}_{3}$ and $H_{4}$. Figure $1(\mathrm{~b})-(\mathrm{d})$ depicts the sound speed profiles of static water and dynamic water (cold- and warm-core eddies). It can be seen that the sound speed is inhomogeneous and in particular that the isospeed lines in the cold- and warm-core eddies are bent in opposite directions. The current profiles of the cold- and warm-core eddies are given in Figure 1(e) and (f). Similarly, the currents in both eddies are inhomogeneous and have opposite directions.
Zone 1 with a range of $4000 \mathrm{~m}$ in Figure 1(a) is discussed in detail for three cases: static water, cold-core eddy, and homogeneous water with a sound speed of $1500 \mathrm{~m} / \mathrm{s}$. The amplitude of the reflections, the traveltime and the seismic imaging are calculated, and the source is located at (2000, $50 \mathrm{~m})$.

As the seismic wave travels through the cold-core eddy, the inhomogeneous velocities of the cold-core eddy cause weak reflected waves (Figure 2). The amplitude of these weak reflected waves is of the order of $10^{-4}$, while the amplitude of the reflections from the reflectors below the water is of the order of $10^{-2}$. The amplitude of reflections from the static water is of the same order of magnitude as that from the cold-core eddy. This demonstrates that the impedance of water is much lower than that of sediment.

The traveltime of reflections from $H_{1}$ for static water and the cold-core eddy is different. For instance, at zero offset, the difference of traveltime between static and homogeneous water is $9.6 \mathrm{~ms}$, while the difference of traveltime between the cold-core eddy and homogeneous water is $20 \mathrm{~ms}$. It can be determined that inhomogeneous water can perturb the traveltime of seismic waves and with the increase of the heterogeneity, this perturbation becomes larger.

Using pre-stack reverse time depth migration, we obtain the migrated images of the four flat reflectors $\mathrm{H}_{1}, \mathrm{H}_{2}, \mathrm{H}_{3}$ and $H_{4}$ (Figure 3 ). An analysis of the migrated images revealed that the four reflectors are positioned incorrectly owing to the perturbation of the inhomogeneous water and that the depth error is proportional to the migration velocity [15]:

$$
\Delta d=v_{\mathrm{m}} \cdot \Delta t / 2
$$

where $\Delta d=d_{i h}-d_{h}, \Delta t=t_{i h}-t_{h}$, and $d_{i h}\left(d_{h}\right)$ is the depth of the reflectors for inhomogeneous (homogeneous) water. $t_{i h}\left(t_{h}\right)$ is the traveltime of reflections from the reflectors for inhomogeneous (homogeneous) water at zero offset. $v_{\mathrm{m}}$ is the migration velocity below the reflector. Eq. (2) is valid under the assumptions of homogeneous media below the water, small offset angle (less than $10^{\circ}$ ), and small-curvature reflectors.

If the traveltime at zero offset for static water (mesoscale eddies) is $t_{s}\left(t_{e}\right)$, the depth error can be calculated by eq. (2). If $\Delta t_{s}=t_{s}-t_{h}, \Delta t_{e}=t_{e}-t_{h}$, and $\Delta t_{e s}=t_{e}-t_{s}$, we have $\Delta t_{e}=\Delta t_{s}+\Delta t_{e s}$, which will be used in the following section.

To study more clearly the effect of inhomogeneous water, we expand the research region to the affected area of the cold-core eddy $(300 \mathrm{~km})$ and then calculate the zero-offset seismic profiling of $H_{1}$ for homogeneous water, static water and the cold-core eddy (Figure 4). By comparing Figure 4(a) and (b) we can establish that the static water causes a delay in the traveltime of reflections from $H_{1}$ across the entire region. Based on eq. (2), we find that this can cause the depth of $H_{1}$ to move down horizontally, but the structure of the flat reflector $H_{1}$ exhibits no change. Comparing Figure 4(a) 


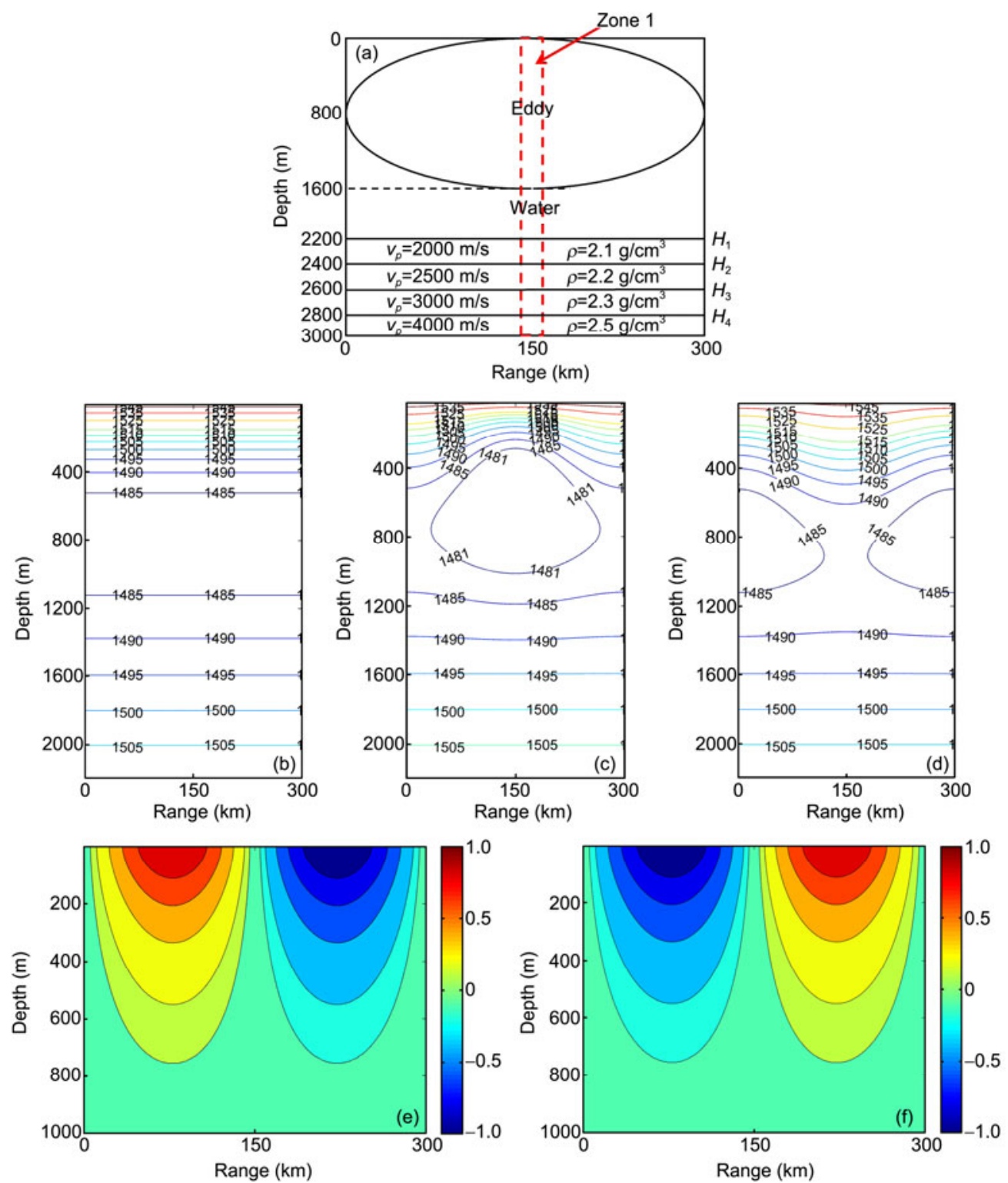

Figure 1 (a) Physical model. Sound speed profile of inhomogeneous water: (b) static water; (c) cold-core eddy; (d) warm-core eddy. Current profile: (e) cold-core eddy; (f) warm-core eddy.

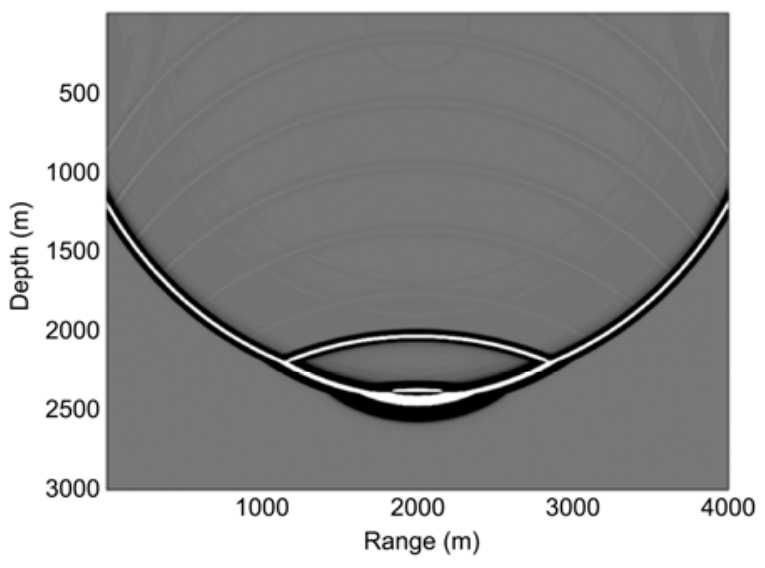

Figure 2 Wavefields around the center of the cold-core eddy at time $1.512 \mathrm{~s}$.

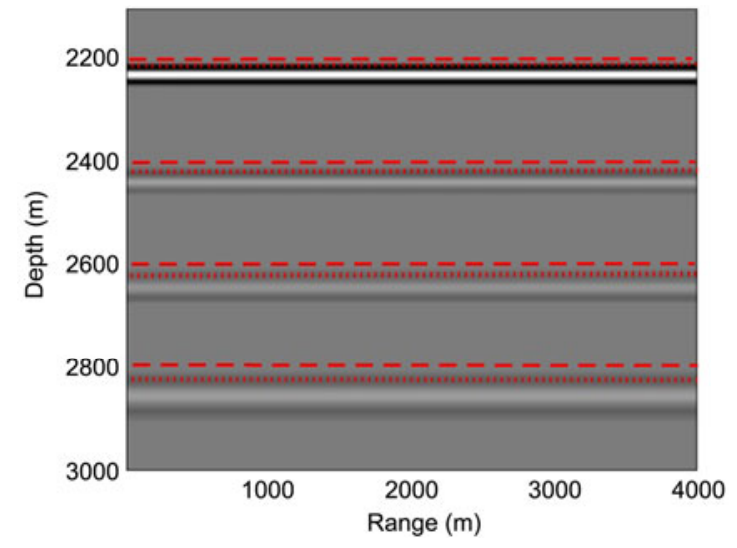

Figure 3 Images migrated with the physical model in Figure 1(a) for cold-core eddy case. The dashed and dotted lines represent the true position of the reflectors and the position of reflectors for static water, respectively. 

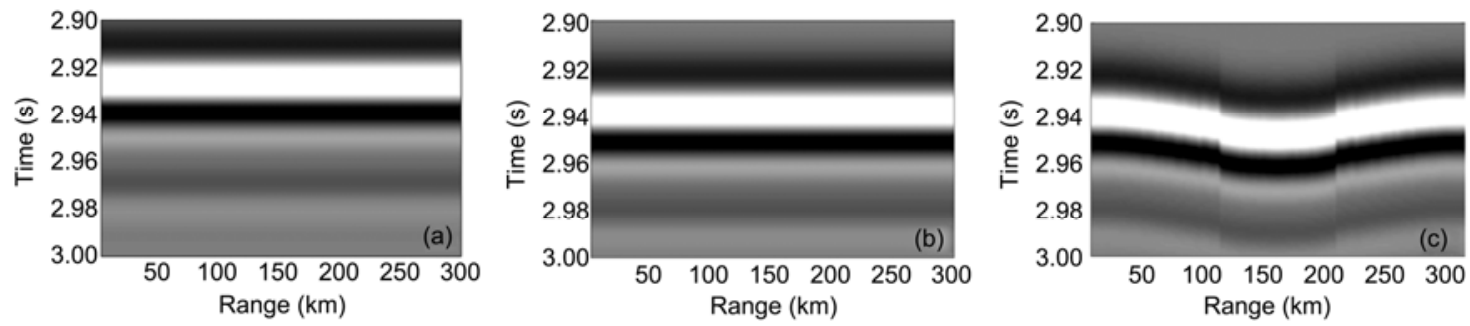

Figure 4 Zero-offset seismic profile of reflector $H_{1}$ : (a) homogeneous water; (b) static water; (c) cold-core eddy.

and (c), we find that the flat reflector $H_{1}$ becomes distorted, and additionally it is positioned incorrectly. Thus, it can be concluded that inhomogeneous water can affect seismic imaging.

Finally, we investigate the difference of the effect between the cold- and warm-core eddies. Figure 5 presents the description of $\Delta t_{e s}$ for the cold-core eddy and the warm-core eddy. A striking feature is that the effect on seismic imaging of the cold-core eddy is the opposite to that of the warmcore eddy. The seismic wave propagates more slowly because of the perturbation of the cold-core eddy, whereas it propagates faster in the presence of the warm-core eddy. Thus, the depth of the reflector is shallower than the real depth of the reflector in the perturbation of the cold-core eddy and it is deeper than the real depth of the reflector in the perturbation of the warm-core eddy.

\section{What causes the influence of the inhomoge- neous water on seismic imaging?}

The above research demonstrates that the structure of horizontal reflectors below the eddy is distorted, and thus the distortion of seismic imaging must be related to the characteristics of the eddy. Henrick et al. [17] highlighted that the effective radius $r_{0}$ and the maximum surface current speed $U_{0}$ are two important characteristic quantities of the eddy model. Thus, in the following section we will study how the distortion of seismic imaging changes with $r_{0}$ and $U_{0}$.

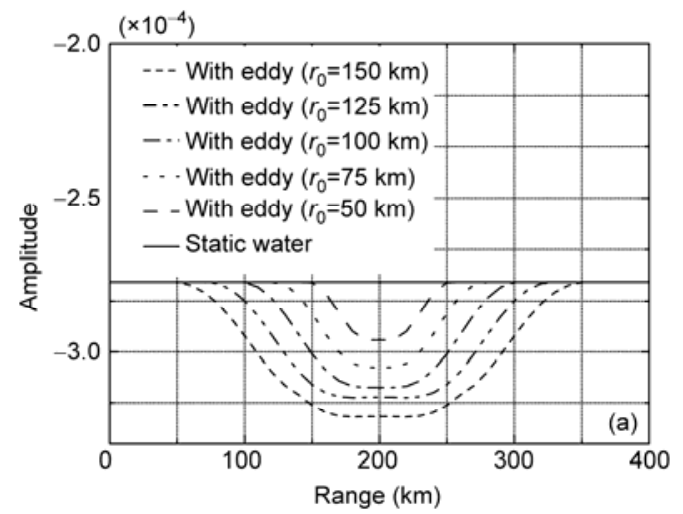

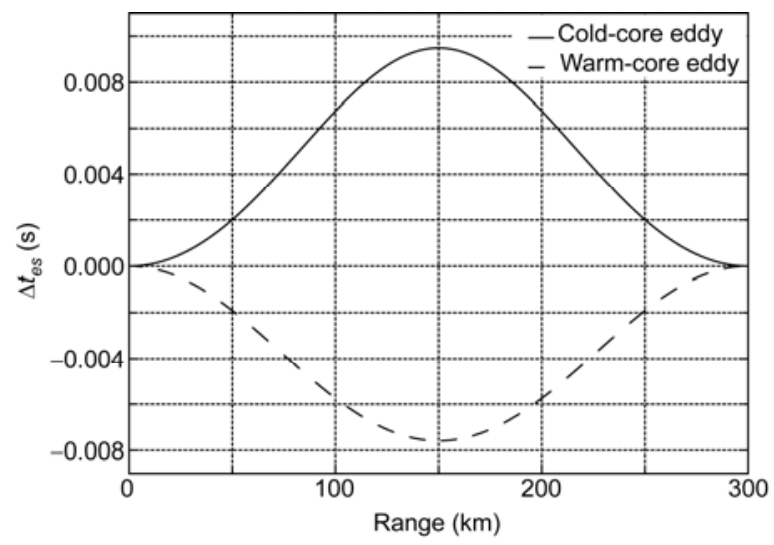

Figure $5 \Delta t_{e s}$ of the cold- and warm-core eddy.

The region we simulate is $400 \mathrm{~km}$ long and the center of the eddy is located at the range of $200 \mathrm{~km}$. Combined with seismic simulation methods, the parabolic equation method (PE method) [18] is used to calculate pressures caused by the reflected wave from the target stratum for different $r_{0}$ and $U_{0}$ (Figure 6). The lines of pressure for static water can reflect the stratum's structure very well; however, with the increasing of $r_{0}$ and $U_{0}$, the distortion of the seismic imaging becomes larger.

Now we introduce the concept of the distorted rate, which means the relative change rate of the pressure caused by the reflected wave of the target stratum in the perturbation of dynamic water. The effective intensity of eddies means the effective radius $r_{0}$ times the maximum surface

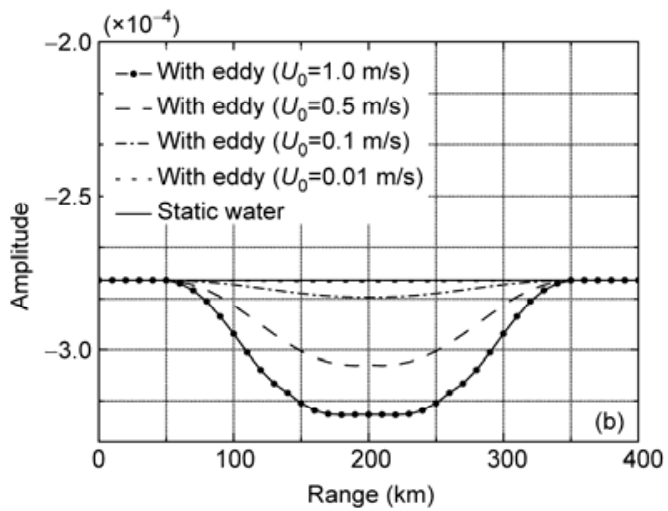

Figure 6 Pressures caused by reflected wave of the target stratum for eddies with different $r_{0}$ (a) and $U_{0}$ (b). 
current speed $U_{0}$; we denote this by $\gamma$ with the unit of 100 $\mathrm{km} \mathrm{m} / \mathrm{s}$. Three typical cases are studied, which have eddies with effective intensities $\gamma$ of $1.5,0.15$, and 0.015 . Considering the symmetrical characteristic of the eddy, here we only consider the distorted rates in the region of $200 \mathrm{~km}$. This region is divided into three intervals (interval 1: 100$200 \mathrm{~km}$, interval 2: 70-100 km and interval 3: 50-70 km) by the distorted rate. Table 2 lists the average distorted rate for eddies with different effective intensities. It is evident that for the same eddy, the distorted rate in different intervals differs considerably.

Studies in seismic oceanography have shown that temperature is an important factor affecting seismic imaging. Therefore, the mechanism of distortions is analyzed using the temperature profile of the eddy. Figure 7 shows the contours of temperature difference caused by eddies with $\gamma$ of 1.5 and 0.015 . The temperature difference in this paper is defined as the absolute value of the difference of temperature between dynamic and static water. Similarly, the region of $200 \mathrm{~km}$ is divided into three intervals (interval 1: 100$200 \mathrm{~km}$, interval 2: 70-100 km and interval 3: 50-70 km). It can be seen from Figure 7 that the primary perturbation of temperature is generally observed in the upper region of the eddy. Moreover, the difference of temperature difference between adjacent intervals is almost 10 times, and this variation law of temperature difference is consistent with the change of average distorted rates in Table 1. In particular, the temperature difference of intervals 2 and 3 in Figure 7(b) is smaller than $0.01^{\circ}$. Correspondingly, the average distorted rates in these intervals are lower than $10^{-4}$, and thus the effect of the eddy on seismic imaging can be neglected. The above results show that temperature difference determines the average distorted rate and that the temperature perturbation in the presence of eddies is the basic reason for the distortion of seismic imaging. We can determine further that inhomogeneous water can lead to the distortion of seismic imaging so long as the heterogeneity of its temperature is sufficiently strong.

\section{How can the influence of inhomogeneous water be eliminated from seismic data?}

In a general case, static water is relatively stable and its density and sound speed vary only with depth. Thus, we can use the wave equation method directly to calculate the time perturbation caused by static water, and then estimate the effect of static water on seismic imaging by eq. (2).

The density, current and sound speed of mesoscale eddies are inhomogeneous in both horizontal and vertical directions. Approaching the center of the eddy, the effect of the eddy on seismic imaging becomes much larger. Thus, we need to find an effective method for estimating the effect of eddies on seismic imaging.

First, the PE method is used to calculate the pressure
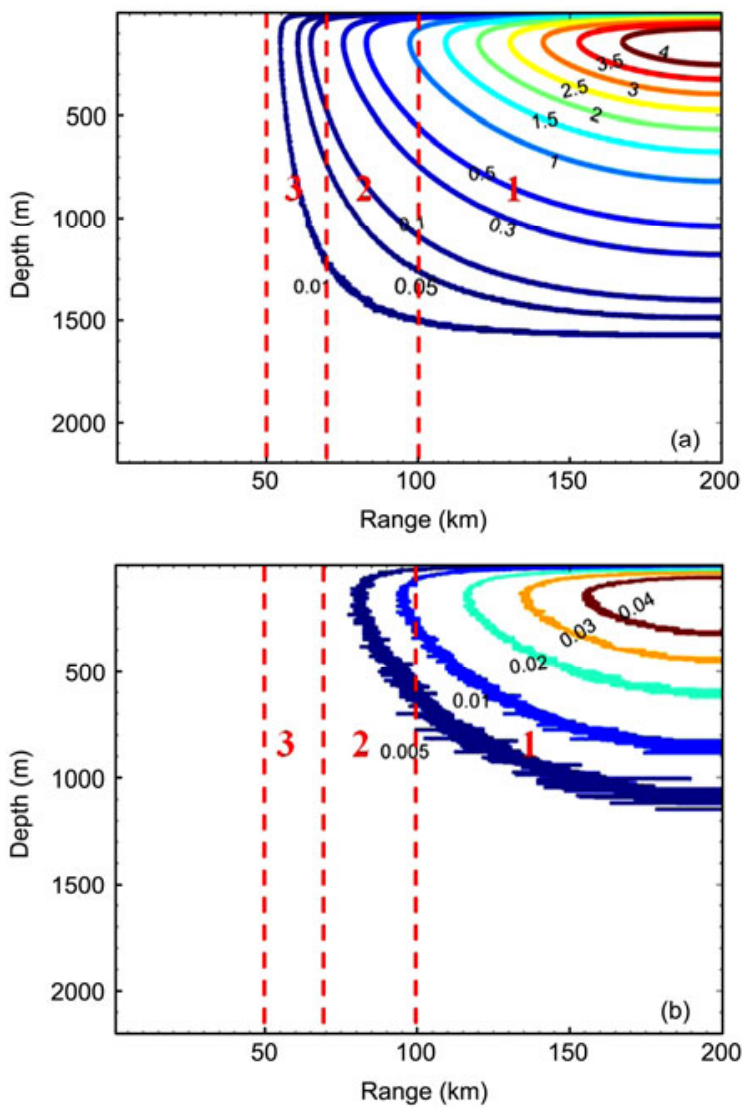

Figure 7 Temperature perturbation contours for eddies with $\gamma$ of 1.5 (a) and 0.015 (b).

Table 1 Average distorted rate for eddies with different effective intensity

\begin{tabular}{clccc}
\hline \multirow{2}{*}{ Interval } & \multirow{2}{*}{$r(\mathrm{~km})$} & \multicolumn{3}{c}{ The average distorted rate } \\
\cline { 3 - 5 } & & \multicolumn{1}{c}{$\gamma=1.5$} & $\gamma=0.15$ & $\gamma=0.015$ \\
\hline 1 & $100-200$ & $7.74 \times 10^{-2}$ & $4.23 \times 10^{-3}$ & $3.71 \mathrm{E} \times 10^{-4}$ \\
2 & $70-100$ & $9.58 \times 10^{-3}$ & $7.70 \times 10^{-4}$ & $6.76 \times 10^{-5}$ \\
3 & $50-70$ & $6.13 \times 10^{-4}$ & $5.41 \times 10^{-5}$ & $4.51 \times 10^{-6}$ \\
\hline
\end{tabular}

perturbation caused by mesoscale eddies. Assuming the water is homogeneous and its depth infinite, we have

$$
\hat{p}_{w}=A\left(\theta_{1}, \theta_{2}, r, z\right) \mathrm{e}^{i\left[B\left(\theta_{1}, \theta_{2}, r, z\right)+\frac{k_{0}}{2}\left(\tilde{n}^{2}-1\right) r\right]},
$$

where $A$ and $B$ are given in [19], $k_{0}$ is the reference wave number, $\tilde{n}=\frac{c_{0}}{\tilde{c}}, \tilde{c} \approx c_{e}+u_{0}, c_{0}$ is $1500 \mathrm{~m} / \mathrm{s}, r$ is the horizontal distance from the source, $z$ is measured positively downward from the sea surface, and $u_{0}$ is defined as above. Denoting the distorted rate caused by eddies as $\Delta D$, we have

$$
\Delta D=a b s\left[\mathrm{e}^{i \frac{k_{0}}{2}\left(\tilde{n}^{2}-1\right) r} / \mathrm{e}^{i \frac{k_{0}}{2}\left(n^{2}-1\right) r}-1\right] \approx a b s\left[\mathrm{e}^{i r k_{0} \frac{\Delta c}{c_{0}}}-1\right],
$$


Table 2 Maximum distorted rates and time perturbation rates for eddies with different effective intensities

\begin{tabular}{ccc}
\hline$\gamma$ & $\Delta D_{t}^{\max }$ & $\Delta D^{\max }$ \\
\hline 0.5 & 0.0009 & 0.0330 \\
0.75 & 0.0014 & 0.0557 \\
1.0 & 0.0018 & 0.0818 \\
1.5 & 0.0026 & 0.1424 \\
2.25 & 0.0037 & 0.2470 \\
\hline
\end{tabular}

where $n=c_{0} / c_{\mathrm{s}}, c_{0}$ is defined as above, $c_{\mathrm{s}}$ is the sound speed of static water, and $\Delta c$ is the difference of sound speed between static water and mesoscale eddies.

Second, we define the time perturbation rate as the relative change rate of the traveltime in the perturbation of dynamic water. Denoting the time perturbation rate caused by eddies as $\Delta D_{t}$, we obtain ( $H$ is the depth of water):

$$
\Delta D_{t}=\Delta t_{e s} /\left(H / c_{\mathrm{s}}\right) \approx\left[H / c_{\mathrm{s}}-H /\left(c_{s}+\Delta c\right)\right] /\left(H / c_{\mathrm{s}}\right) \approx \frac{\Delta c}{c_{0}} .
$$

By comparing eq. (4) with eq. (5), we found that $\Delta D$ and $\Delta D_{t}$ are determined by the sound speed perturbation caused by eddies. $\Delta D$ contains the information about pressure, whereas $\Delta D_{t}$ contains information about phase, which is the key reason for the depth error of migrated images. If we can calculate $\Delta D_{t}$ using $\Delta D$, the computational efficiency can be improved greatly. Denoting $\Delta D$ and $\Delta D_{t}$ at the center of the eddy as $\Delta D^{\max }$ and $\Delta D_{t}^{\max }$, we calculate $\Delta D^{\max }$ and $\Delta D_{t}^{\max }$ of the eddies with different effective intensities at the frequency of $25 \mathrm{~Hz}$ (Table 2). It should be noted that $\Delta D^{\max }$ could be computed by effective intensity of the eddy [18].

Applying the fitting method to Table 2, we determined the quantitative relationship between $\Delta D^{\max }$ and $\Delta D_{t}^{\max }$ as follows (SD-TD relationship):

$$
\begin{aligned}
\Delta D_{t}^{\max }= & 0.01\left(0.0136+2.3713 \times \Delta D^{\max }-5.3334 \times\left(\Delta D^{\max }\right)^{2}\right. \\
& \left.+6.4205 \times\left(\Delta D^{\max }\right)^{3}\right) .
\end{aligned}
$$

To test the accuracy of the SD-TD relationship, two eddies with effective intensities of 1.56 and 1.78 are chosen arbitrarily. Figure 8 shows that the error of $\Delta D_{t}^{\max }$ calculated by the SD-TD relationship is less than $10^{-4}$. It is worthwhile emphasizing that eq. (6) is calculated at the frequency of $25 \mathrm{~Hz}$.

The time perturbation at the center of the eddy is calculated above, and in the following, we will consider the time perturbation at an arbitrary point of the eddy. Using $\Delta D_{t}^{\max }$ and a linear approximation, we have

$$
\Delta t_{e}=\frac{\Delta D_{t}^{\max } \times t_{s}}{r_{0}}\left(r_{0}-r^{\prime}\right)+\Delta t_{s},
$$

where $r_{0}, t_{s}$ and $t_{e}$ are defined as above, and $r^{\prime}$ is the horizontal distance from the center of the eddy. Thus, we can use eqs. (6), (7) and (2) to estimate the effect of the eddy on seismic imaging. Similarly, we can estimate the effect of other kinds of inhomogeneous water on seismic imaging. Figure 9 gives the definitive method of operation.

We have answered the three questions raised at the beginning of the paper. (1) The effect of inhomogeneous water on seismic imaging varies with the heterogeneity of the

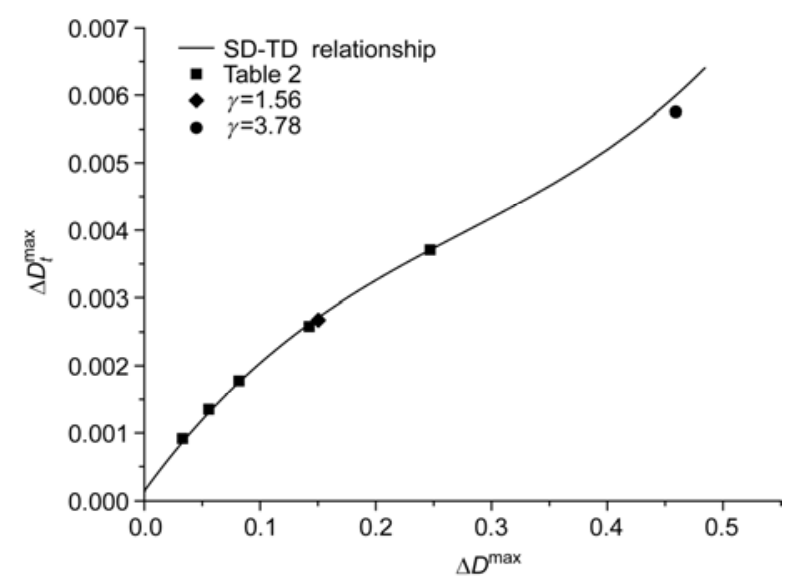

Figure 8 Verification of SD-TD relationship.

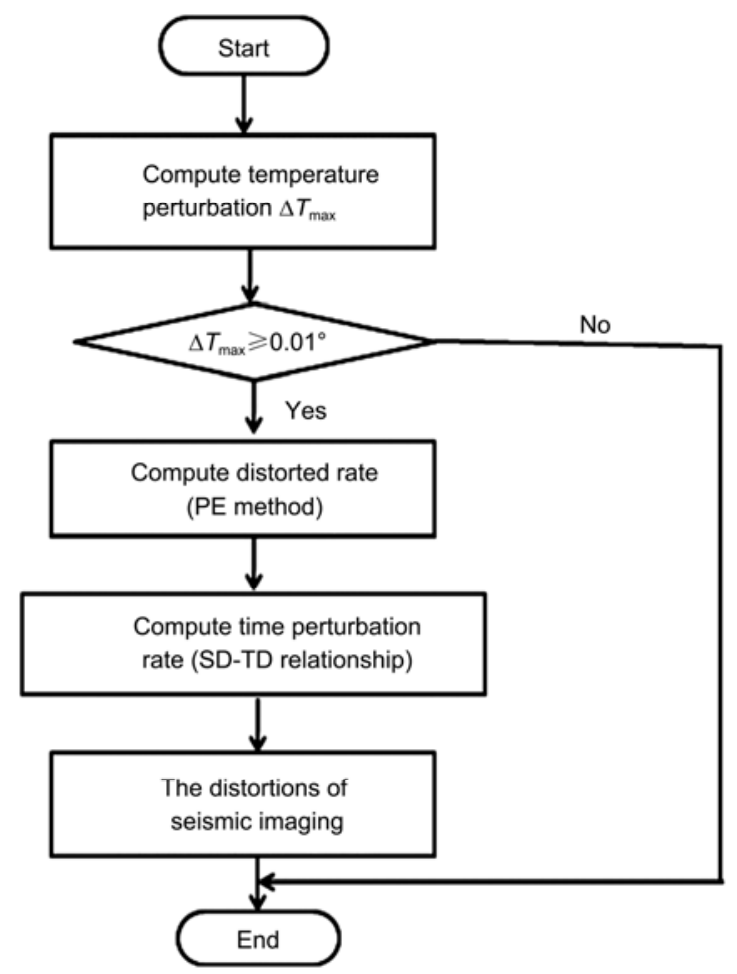

Figure 9 Definitive method of operation for estimating the distortion of seismic imaging. 
water: static water only causes depth errors in migrated images; mesoscale eddies do not only cause depth errors, but also distort the migrated images. (2) The heterogeneity of water temperature is the primary cause of the distortions. (3) The distortions of seismic imaging caused by inhomogeneous water can be eliminated from seismic data.

This work was supported by the National Natural Science Foundation of China (41074058, 41074097 and 41210005) and the National Basic Research Program of China (2009CB219405-1).

1 Ruddick B. Sounding out ocean fine structure. Science, 2003, 301: $772-773$

2 Krahmann G, Brandt P, Klaeschen D, et al. Mid-depth internal wave energy off the Iberian Peninsula estimated from seismic reflection data. J Geophys Res, 2008, 113: C12016

3 Song H B, Luis P, Wang D X, et al. Seismic images of ocean meso-scale eddies and internal waves (in Chinese). Chin J Geophys, 2009, 52: 2775-2780

4 Pinheiro L M, Song H, Ruddick B, et al. Detailed 2-D imaging of the Mediterranean outflow and meddies off $\mathrm{W}$ Iberia from multichannel seismic data. J Mar Syst, 2010, 79: 89-100

5 Wood W T, Holbrook W S, Sen M K, et al. Full wave form inversion of reflection seismic data for ocean temperature profiles. Geophys Res Lett, 2008, 35: L04608

6 Song H B, Dong C Z, Chen L, et al. Reflection seismic methods for studying physical oceanography: Introduction of seismic, oceanography (in Chinese). Progr Geophys, 2008, 23: 1156-1164

7 Gonella J, Michon D. Deep internal waves measured by seismicreflection within the Eastern Atlantic water mass. Cr Acad Sci II, 1988, 306: 781-787

8 Phillips J D, Dean D F. Multichannel Acoustic Reflection Profiling of
Ocean Watermass Temperature/Salinity Interfaces. New York: Springer, 1991. 199-214

9 Holbrook W S, Paramo P, Pearse S, et al. Thermohaline fine structure in an oceanographic front from seismic reflection profiling. Science, 2003, 301: 821-824

10 Nandi P, Holbrook W S, Pearse S, et al. Seismic reflection imaging of water mass boundaries in the Norwegian Sea. Geophys Res Lett, 2004, 31: L23311

11 Nakamura Y, Noguchi T, Tsuji T, et al. Simultaneous seismic reflection and physical oceanographic observations of oceanic fine structure in the Kuroshio extension front. Geophys Res Lett, 2006, 33: L23605

12 Biescas B, Sallare S V, Pelegr J L, et al. Imaging meddy fine structure using multichannel seismic reflection data. Geophys Res Lett, 2008, 35: L11609

13 Cheng X H, Qi Y Q, Wang W Q. Seasonal and interannual variabilities of mesoscale eddies in South China Sea (in Chinese). J Trop Oceanogr, 2005, 24: 51-59

14 Guo Y J, Fang W D, Fang G H, et al. Temporal and spatial variation characteristics of the surface circulation in the South China Sea based on eleven-year altimeter data (in Chinese). Chin Sci Bull (Chin Ver), 2006, 51: 1-8

15 Ji L L, Lin M. Numerical analysis of the effect of mesoscale eddies on seismic imaging. Pure Appl Geophys, 2013, 170: 259-270

16 Kormann J, Cobo P, Prieto A. Perfectly matched layers for modelling seismic oceanography experiments. J Sound Vib, 2008, 317: 354-365

17 Henrick R F, Siegmann W L, Jacobson M J. General analysis of ocean eddy effects for sound transmission applications. J Acoust Soc Am, 1977, 62: 860-870

$18 \mathrm{Ji}$ L L, Lin M. The distortion of seismic imaging in the presence of mesoscale eddies and analysis of influencing factors (in Chinese). Chin J Geophys, 2013, 56: 195-203

19 Ji L L. Numerical analysis of the effect of mesoscale eddies on seismic imaging (in Chinese). Dissertation for the Doctoral Degree. Beijing: Graduate University of Chinese Academy of Sciences, 2012

Open Access This article is distributed under the terms of the Creative Commons Attribution License which permits any use, distribution, and reproduction in any medium, provided the original author(s) and source are credited. 